\title{
Horizontal Field Generated by Lightning with the Surface Impedance Method
}

\author{
Wei $\mathrm{HE}^{1 *}$, Jiajia $\mathrm{HE}^{2}$, Ping $\mathrm{LU}^{1}$ \\ 1 School of Physics and Electronic Information, Yunnan Normal University, Kunming, China
}

2 School of Physics and Electronic Information, Shanxi Normal University, Xi'an, China

Corresponding author: Wei HE, email: he99wei@aliyun.com

Keywords: electromagnetic field; horizontal field; surface impedance method

\begin{abstract}
The lightning produces a powerful lightning current, and it caused the electromagnetic fields, the optical radiation, the shock wave and the thunder and so on physical effects. Electromagnetic fields generated by lightning are divided into horizontal electric field and vertical electric field. The accurate calculation of the electromagnetic field generated by lightning has an important role in lightning research work. In this paper, a new method by using the expression for the surface impedance of the earth with finite conductivity was presented, and the results of horizontal field calculated from wave tilt and surface impedance with exact expression is compared. The new method is suit for calculating horizontal field generated by return strokes at distances as close as $200 \mathrm{~m}$, while these close ranges the use of the wave tilt expression can cause large errors.
\end{abstract}

\section{Introduction}

The experimental findings generated by lightning field component parallel to the ground, that the horizontal field of overhead power lines would play a decisive role influence[1] [2]. Therefore, to determine the voltage induced in the power line, the first accurately calculating the return stroke of lightning generated horizontal field, Sommerfeld[2] use of the finite conductivity dipole generated farm on the land to calculate the horizontal field, and he had given a series of integral equations. These final results obtained by solving the integral equation. But solving the process takes a lot of computing time, and that may be encountered if the integrand convergence problems. In order to avoid spending a lot of computing time, we used for solving the horizontal field of approximate method for the ramp method[4] [5]. This method assumes that when a plane electromagnetic wave propagation in a limited conductive ground, the electric field is tilted to the ground (if the earth was considered fully conductor, the electric field was perpendicular to the ground surface), I.e., the component parallel to the ground electric field is not zero, and its size is determined by the conductivity and dielectric constant of the earth. And its expression is:

$$
\frac{E_{h}(j \omega)}{E_{Z}(j \omega)}=\frac{1}{\sqrt{\varepsilon+\frac{\sigma}{j \omega \varepsilon_{0}}}}
$$

By the formula (1) to find the horizontal component of the electric field, it may meet two conditions: the radiation field, and the rake graze incident. The following discussion, only in distant places just to meet these two conditions, we use it to seek the lightning channel closer from the horizontal field. So it would produce large errors. Therefore, this paper introduces a new method to calculate the level field. This method is used the earth's surface impedance to solve the lightning electromagnetic field, namely:

$$
\frac{E_{k}(j \omega)}{B_{p}(j \omega)}=\frac{c}{\sqrt{\varepsilon+\frac{\sigma}{j \omega \varepsilon_{0}}}}
$$

Later we will discuss the horizontal field by the formula (2) to calculate the vicinity of the lightning channel, which is much more precise than by the formula (1), and easy to solve. 


\section{Perpendicular dipole electromagnetic field}

Consider the case shown in Figure 1. $\mathrm{H}$ is the dipole height from the ground, The direction of the current dipole in the z-axis direction. The assuming that of the earth is niform and the surface is flat, according to [6], the electromagnetic field $\mathrm{P}$ point is:

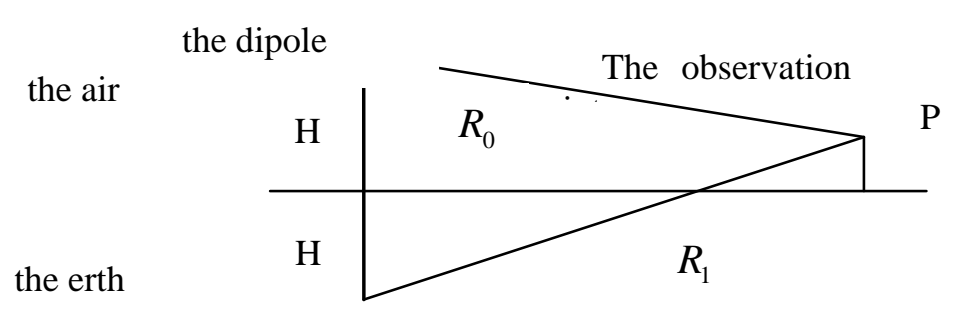

Figure 1 the dipole on a limited conductive ground

$$
\begin{aligned}
& E_{h}(\mathrm{j} \omega)=\frac{M}{4 \pi \varepsilon_{0}(j \omega)}\left\{\left(3+3 \gamma_{0} R_{0}+\gamma_{0}^{2} R_{0}^{2}\right)\right\} \times \sin \psi_{0} \cos \psi_{0} \frac{e^{-\gamma_{0} R_{0}}}{R_{0}^{3}}+\left(3+3 \gamma_{0} R_{1}+\rho \gamma_{0}^{2} R_{1}^{2}\right) \\
& \sin \psi_{1} \cos \psi_{1} \times \frac{e^{-\gamma_{0} R_{1}}}{R_{1}^{3}}+\frac{2}{n}\left(3+3 \gamma_{0} R_{1}+\rho \gamma_{0}^{2} R_{1}^{2}\right) \sin \psi_{1} \cos \psi_{1} \times \frac{e^{-\gamma_{0} R_{1}}}{R_{1}^{3}}+ \\
& \left.\gamma_{0}^{2}\left[\frac{\cos \psi_{2} e^{-\gamma_{0} R_{2}}}{R_{2}+d+z+H}-\frac{\cos \psi_{1} e^{-\gamma_{0} R_{1}}}{R_{1}+z+H}\left(1+\gamma_{0} d\right)\right]+2 \Delta\left[1-\left[\frac{1-\rho}{2}\right] F(\omega)\right] \cos \psi_{1}\left(\psi_{0}^{2} R_{1}^{2}\right) \frac{e^{-\gamma_{0} R_{1}}}{R_{1}^{3}}\right\} \\
& E_{z}(j \omega)=-\frac{M}{4 \pi \varepsilon_{0}(j \omega)}\left\{\left[\left(1-3 \sin ^{2} \psi_{0}\right)\left(1+\gamma_{0} R_{0}\right)+\gamma_{0}^{2} R_{0}^{2} \cos ^{2} \psi_{0}\right] \frac{e^{-\gamma_{0} R_{0}}}{R_{0}^{3}}\right. \\
& \left.\left[\left(1-3 \sin ^{2} \psi_{1}\right)\left(1+\gamma_{0} R_{1}\right)+\rho \gamma_{0}^{2} R_{1}^{2} \cos ^{2} \psi_{1}\right] \frac{e^{-\gamma_{0} R_{0}}}{R_{1}^{3}}+(1+\rho) F(\omega) \cos ^{2} \psi_{1}\left(\gamma_{0}^{2} R_{1}^{2}\right) \frac{e^{-\gamma_{0} R_{1}}}{R_{1}^{3}}\right\} \\
& B_{p}(j \omega)=\frac{\mu_{0}}{4 \pi}\left\{\left(1++\gamma_{0} R_{0}\right) \cos \psi_{0} \frac{e^{-\gamma_{0} R_{0}}}{R_{0}^{3}}+\left(1+\rho \gamma_{0} R_{1}\right) \cos \psi_{1} \frac{e^{-\gamma_{0} R_{1}}}{R_{1}^{2}}+\right. \\
& \left.(1+\rho) \times F(\omega) \cos \psi_{1}\left(\gamma_{0} R_{1}\right) \frac{e^{-\gamma_{0} R_{1}}}{R_{1}^{2}}\right\}
\end{aligned}
$$

According to the literature [6], long as it satisfies[6] $n^{2}>>1$, the formula (3) to (5) on the ground anywhere are accurate and effective targeting. The angular frequency range of electromagnetic field generated lightning is $10^{4} \sim 10^{7} \mathrm{rad} / \mathrm{s}$. Thus, equation (3) to (5) are used to calculate a ground electromagnetic fields generated lightning with the finite conductivity strictly.

\section{Comparison of the ramp method with the surface impedance method}

$E_{h w}(j \omega), E_{h s}(j \omega)$, and the $E_{h}(j \omega)$ were represented by the formula (1), and the formula (2) and (3) were used to calculate the horizontal electric field. The results were shown in Figure 2 to Figure 4. 


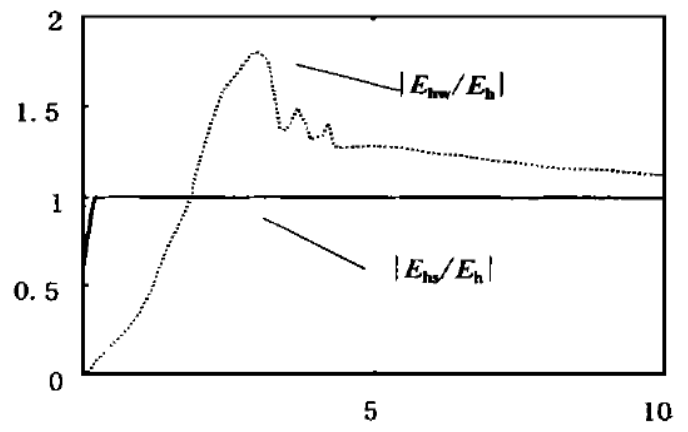

The distance $/ \mathrm{km}$

(a) $\sigma=0.01 \mathrm{~m} / \mathrm{s}$

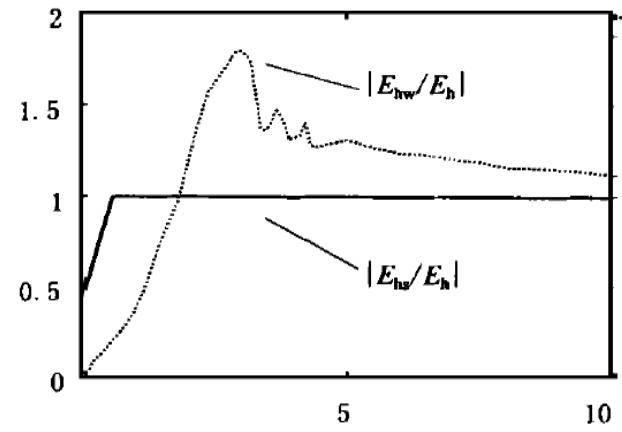

The distance $/ \mathrm{km}$

(b) $\sigma=0.001 \mathrm{~m} / \mathrm{s}$

Figure 2 as a function of distance from the dipole in the ground

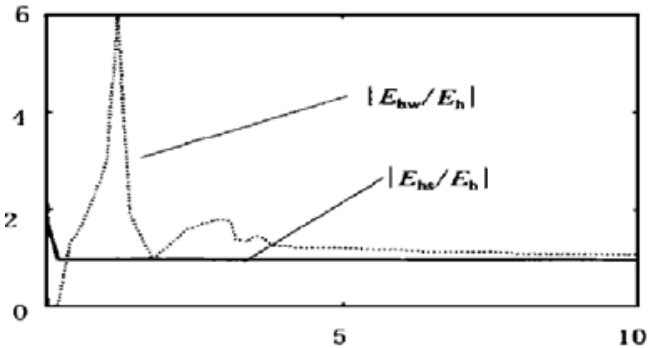

The distance $/ \mathrm{km}$

(a)

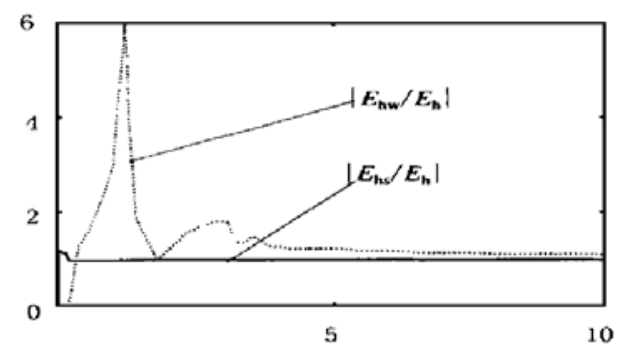

The distance $/ \mathrm{km}$

(b)

Figure 3 the dipole above the ground at a height of case 500m (With the other parameters the same as in Fig. 2)

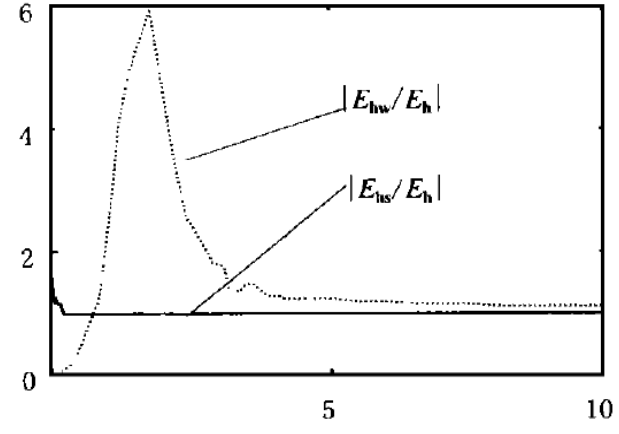

The distance $/ \mathrm{km}$

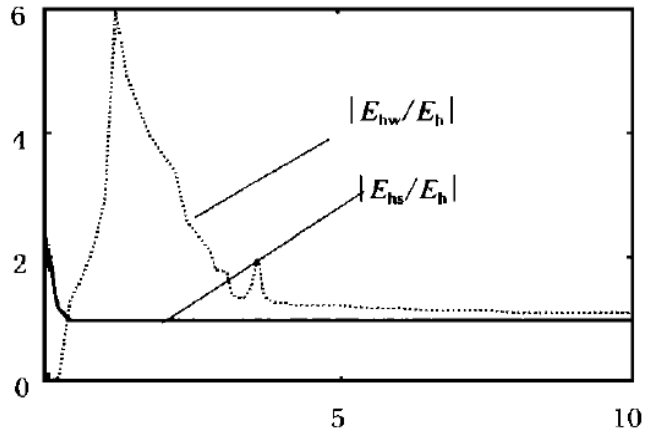

The distance $/ \mathrm{km}$

Figure 4 dipoles in the ground at the height of the case $1000 \mathrm{~m}$ (With the other parameters the same as in Figure 2)

The main problem we are interested in that the distribution of lightning electromagnetic pulse starts to the maximum peak of this time. Because this time the di / dt is maximum, at this time lightning to produce induced voltage overhead lines. This induced voltage mainly depends on the level electric field. During this time, the lightning hits back to flow path length to be not exceed $10000 \mathrm{~m}$, and its frequency of the electromagnetic fields is nearly $10^{5} \mathrm{rad} / \mathrm{s}$ [7] . That is in line with the case discussed above. In this case, the lightning electromagnetic field horizontal component is calculated on a limited conductive ground generated by surface impedance method, which is very accurate. Especially when a distance of the observation point and the lightning channel is greater than $200 \mathrm{~m}$, the calculation result is the same solved by this method exactly.

\section{Conclusions}

When lightning discharges to the ground, the horizontal component of the current comeback in the electromagnetic field generated by lightning at the limited conductive ground, it can be 
calculated by the surface impedance of the earth. As a result, the accuracy is high. This method is particularly applicable to the observation point to the channel a distance greater than the case of a lightning strike of $200 \mathrm{~m}$. If the distance is less than $200 \mathrm{~m}$, this method can also cause the error. At this time we only solved exactly by the formula (3).

\section{Acknowledgment}

This project was supported by National Natural Science programs (50367001, 51267021) and Yunnan Province Science and Technology bureau program (2009ZC055M).

\section{References}

[1] $\mathrm{M}$ asterM J, UmanM A. Lightning-induced voltages on power line: theory. IEEE Trans PowerApparSyst, 1984, 103: 2 502 2518

[2] Rachidi F, NucciC A, IanozM. Influence of a lossy ground on lightning-induced voltages on overhead lines. IEEE Trans Electromagn Compat, 1996, 38: 250 264

[3] Sommerfeld A. Partial differential equations in physics. New York: Academ ic, 1949

[4] Diendorfer G. Induced voltage on an overhead line due to nearby lightning. IEEE Trans Electromagn Compat, 1990, 32: 292 299

[5] Vance E F. Coupling to shielded cables. New York: Weiley. 1978

[6] UmanM A, M cLain D K, Krider E P. The electromagnetic radiation from a finite antenna. Amer, JPhy, 1975, 43: 33 38

[7] Chowdhuri P, G ross E T B, Voltage surges induced on overhead lines by lightning strokes. Proc Inst Elec Eng, 1967, 114: 1 899 1 907 